\title{
Apresentação da Prática de Investigação Científica Envolvendo Distúrbios Osteometabólicos para Alunos do Ensino Médio
}

\author{
Scientific Investigations and Practices Associated to \\ Osteometabolic Disturbes for High Schools
}

\section{RESUMO}

A ciência é a aquisição de conhecimento sobre a natureza e as atividades humanas. A prática da pesquisa por alunos do ensino médio é um recurso educativo dinâmico e efetivo pautado na investigação. $\mathrm{O}$ objetivo do trabalho foi aproximar esses alunos da pesquisa e despertar o espírito investigativo através do conhecimento de metodologias para o estudo de distúrbios osteometabólicos e as aplicações deles na vida da população, compartilhando o conhecimento na forma de atividade de extensão. Escolas de Ribeirão Preto-SP foram convidadas para visitarem o Laboratório de Análises Morfológicas da Faculdade de Odontologia de Ribeirão Preto e o Laboratório de Bioengenharia da Faculdade de Medicina de Ribeirão Preto. Quarenta alunos conheceram a infraestrutura desses laboratórios, manusearam equipamentos, aprenderam sobre as etapas das pesquisas e os aspectos éticos associados ao uso de animais. $\mathrm{O}$ trabalho foi parabenizado pelo intercâmbio com os alunos e pelas explicações simples e claras, mesmo se tratando de questões técnicas da bioengenharia e das ciências da saúde. Os alunos relataram que gostaram da visita por manusearem os equipamentos e verem como funciona a investigação científica. A visita funcionou como mecanismo de fortalecimento do elo entre a universidade e a sociedade ao mostrar que a investigação científica é agente transformador da realidade social.

Palavras-chave: Investigação Científica. Escolas. Distúrbios Osteometabólicos.

\begin{abstract}
Science is the acquisition of knowledge about nature and human activities. Research practice for high school students is a dynamic and effective educational resource supported in research. The objective of this work was to bring these students close to research activities and awaken the spirit of investigation through knowledge of methodologies for the study of osteometabolic disorders and their applications for
\end{abstract}

Taiane Paliologo e Miliane Gonçalves GONZA G A

Universidade de São Paulo. Faculdade de Medicina de Ribeirão Preto, São Paulo, Brasil

\section{Joño Paulo}

MARDEgan IsSA E

Vinícius Pedrazzi

Universidade de São Paulo. Faculdade de Odontologia de Ribeirão Preto, São Paulo, Brasil 
the population, by sharing knowledge, in the form of outreach activity. Schools of Ribeirão Preto, São Paulo were invited to visit the Morphological Laboratory of the Faculty of Dentistry of Ribeirão Preto and Bioengineering Laboratory, Faculty of Medicine of Ribeirão Preto. Forty students knew the infrastructure of these laboratories, equipment management, learned about the stages of the research and the ethical issues associated to animals' use. These activities were congratulated regarding to students exchange and the supplied explanations, simple and clear, even when dealing with technical issues of bioengineering and health sciences. Students reported that they enjoyed the visit, mainly by the opportunity to handle the equipment and see how scientific research works. The developed activities represent a mechanism to strengthen the link between the university and society, showing that the scientific research is a transforming agent of social conditions.

Keywords: Scientific Research. Schools. Osteometabolic Disorders.

\section{INTRODUÇÃO}

A palavra "ciência" é definida como um conjunto de conhecimentos adquiridos ou acumulados que possuem um caráter social, histórico e universal, além de serem dotados de objetividade e estruturados com base em teorias, métodos e linguagens próprias. Desta forma, tem como objetivo compreender e orientar a natureza e as atividades humanas [11]. Além disso, a ciência pode ser entendida como um sistema de acomodação temporária baseada na perspectiva de permutação e aplicação dos resultados obtidos na pesquisa [13]. Assim, é constituída como a aquisição de conhecimento sobre a natureza e as atividades humanas, com o objetivo de aumentar e melhorar o conhecimento, descobrindo e explicando novos fatos. A grande aliada da ciência é a pesquisa científica. Nela, o investigador tem o desafio de solucionar o problema através de vários métodos pertinentes ao campo de conhecimento, inclusive através das experimentações.

As pesquisas envolvendo procedimentos experimentais ocuparam papel de destaque na consolidação e estabelecimento das ciências naturais a partir do século XVII, na medida em que as leis formuladas deveriam ser testadas dentro de uma lógica sequencial de formulação de hipóteses e verificação de consistência. Ocorreu, naquele período, uma ruptura com as práticas de investigação vigentes que estavam fortemente impregnadas pelo divino e pelo senso comum. A experimentação ocupou um lugar privilegiado na proposição de uma metodologia científica, que se pautava pela racionalização de procedimentos, tendo assimilado formas de pensamento características como a indução e a dedução [14].

A prática da pesquisa por alunos do ensino médio pode ser vista como princípio educativo, utilizando a investigação como instrumento metodológico para construir o conhecimento [12]. Os projetos investigativos de pesquisa são propostas pedagógicas capazes de criar situações de aprendizagem mais dinâmicas e efetivas pelo questionamento e reflexão, contribuindo para que o aluno participe do seu próprio processo 
de aprendizagem e deixando o ensino mais dinâmico. Pensar e agir cientificamente auxilia o indivíduo entender-se no mundo e com o mundo [16, 20].

A experimentação é capaz de despertar um forte interesse em alunos de diversos níveis de escolarização, atribuindo um caráter motivador, lúdico, essencialmente vinculado aos sentidos, além de aumentar a capacidade de aprendizado, pois funciona como meio de envolver o aluno nos temas que estão em pauta [14].

O objetivo do trabalho foi aproximar o estudante do ensino médio à prática de pesquisa da universidade. Além disso, despertar o espírito crítico investigativo através do conhecimento de metodologias de pesquisa e modelos experimentais para estudo de distúrbios osteometabólicos e as aplicações práticas dessas pesquisas para a melhoria das condições de vida da população como um todo.

\section{MATERIAIS E MÉTODOS}

Escolas do município de Ribeirão Preto, em São Paulo, foram convidadas a participar do trabalho. O contato foi realizado por telefone e e-mail e as visitas foram programadas de acordo com a disponibilidade do calendário escolar e dos laboratórios. Posteriormente, o cronograma da visita foi montado a fim de permitir um melhor aproveitamento pelos alunos através de uma ordem compatível com o transcorrer natural das etapas experimentais. A rotina nos laboratórios a serem visitados foi readequada a fim de permitir a apresentação dos vários procedimentos, enquanto os docentes, discentes e técnicos se organizam para ajudar nas atividades.

Os alunos das escolas que aceitaram o convite, juntamente com os professores, realizaram uma visita ao Laboratório de Análises Morfológicas da Faculdade de Odontologia de Ribeirão Preto e ao Laboratório de Bioengenharia da Faculdade de Medicina de Ribeirão Preto, que pertencem à Universidade de São Paulo.

No Laboratório de Análises Morfológicas os alunos de ensino médio receberam explicações sobre as pesquisas vigentes no laboratório, observaram uma lâmina óssea no microscópio e também puderam realizar um corte no micrótomo. Os alunos receberam também orientações sobre a utilização da balança, a descalcificação óssea, a coloração, a inclusão de parafina, a estufa e o criostato, que são aparelhos usados no cotidiano das pesquisas envolvendo ossos e músculos.

No Laboratório de Bioengenharia os alunos receberam explicações sobre as pesquisas realizadas no local; visitaram o biotério e puderam ver animais usados como modelos experimentais, sendo eles ratos, camundongos e coelhos. As condições de alojamento adequadas e os cuidados necessários para o bem estar dos animais e para o bom andamento das pesquisas foram salientados, assim como as questões éticas pertinentes ao uso de animais em pesquisas científicas. Os equipamentos usados para pesquisas envolvendo atividade física foram vistos, incluindo a esteira elétrica para treinamento de corrida animal e o tanque de água utilizado para natação dos animais. A sala de cirurgia também foi apresentada com os equipamentos e instrumentos usados durante os procedimentos operatórios. Além disso, os alunos puderam conhecer o equipamento para análise da marcha animal, as máquinas de 
análises mecânicas de compressão, torção e fadiga, máquina de impressão $3 \mathrm{D}$ para configuração de prótese ortopédica, máquina de microscopia óptica e a máquina de medição precisa. Alguns pôsteres sobre trabalhos realizados com eletroacupuntura, ultrassom e laser também foram apresentados.

Após a visita aos laboratórios, os alunos receberam a condução do circular interno do campus Ribeirão Preto da Universidade de São Paulo e realizaram uma visita monitorada, observando a estrutura das faculdades, da biblioteca e demais prédios. Após os dias programados para as visitas, os professores que acompanharam seus alunos fizeram relatórios sobre a visita, comentando os aspectos mais relevantes.

\section{RESULTADO}

Duas escolas aceitaram o convite para que seus alunos de ensino médio participassem do trabalho com a realização de uma visita para conhecerem o Laboratório de Análises Morfológicas da Faculdade de Odontologia de Ribeirão Preto e o Laboratório de Bioengenharia da Faculdade de Medicina de Ribeirão Preto nos dias marcados para cada escola. Quarenta alunos compareceram e puderam conhecer a infraestrutura dos dois laboratórios que são diretamente ligados a pesquisas envolvendo desordens musculoesqueléticas, inclusive distúrbios osteometabólicos.

Durante a visita, eles puderam não apenas contemplar, mas manusear alguns equipamentos e aprender como cada etapa do processo de investigação científica é realizada e como os resultados são apresentados em dissertações, artigos e pôsteres. Da mesma forma, foram alertados quanto aos aspectos éticos atrelados às pesquisas que usam o modelo experimental com animais. A importância da investigação científica foi ressaltada, baseando-se principalmente nos objetivos e nas justificativas dos projetos desenvolvidos nos laboratórios envolvendo os distúrbios osteometabólicos. Nos relatórios enviados pelos professores que acompanharam os alunos, o trabalho foi parabenizado pela iniciativa da Universidade de buscar o intercâmbio com os alunos que estão se preparando para pleitear uma vaga no ensino superior ao mostrar como atua no processo de produção de conhecimento e na importância deste. Da mesma forma, foram consideradas a organização da visita e também as explicações em linguagem simples e clara mesmo se tratando de questões técnicas do universo científico. Os alunos relataram que gostaram da visita por poderem manusear equipamentos delicados e por verem como funciona o cotidiano das pessoas que lidam com a pesquisa experimental. Além disso, expressaram o desejo de participar de outros projetos da Universidade.

\section{DISCUSSÃO}

O convite para conhecer os laboratórios e mostrar quais as atividades de pesquisa desenvolvidas quanto aos distúrbios osteometabólicos e suas principais etapas funcionou como uma forma de aproximar a universidade dos alunos de ensino médio, o que constitui um dos principais objetivos das atividades de extensão $[7$, 19, 22, 29, 
30, 31]. A pesquisa sobre esses distúrbios é importante na composição de novos métodos de exames e de intervenções concernentes à reabilitação. Os distúrbios osteometabólicos como osteoporose, hipo ou hipercalcemia são muito comuns na população e comprometem sua qualidade de vida [32]. A sociedade e a universidade devem estabelecer um elo de interação que vise promover a melhoria da qualidade de vida da população por meio de práticas de extensão universitária. A universidade tem o papel de transmitir o conhecimento e também contribuir para que a própria população também possa ser agente do processo de transformação que abrange as perspectivas de inclusão social e de desenvolvimento sustentável [9, 23, 27]. Neste contexto, o desenvolvimento científico e tecnológico promovido principalmente nas universidades é capaz de gerar grandes impactos na expectativa de vida da população e em sua conjuntura socioeconômica [18].

Tal desenvolvimento advém da pesquisa científica, a qual consiste em um processo sistemático que busca construir o conhecimento ao gerar novos conhecimentos ou até mesmo corroborar ou refutar o conhecimento prévio. A pesquisa se pauta em uma série de procedimentos metodológicos que vai desde o planejamento, a execução, até a divulgação dos resultados obtidos, sendo que todas essas etapas devem ser procedidas de maneira condizente com o campo de estudo proposto e de forma que garanta a reprodutibilidade do experimento. No caso da saúde, o foco da pesquisa deve ser direcionado para necessidade das pessoas de acordo com o conceito de saúde estabelecido pela Organização Mundial de Saúde, ou seja, a saúde deve ser vista como o bem estar biopsicossocial do ser humano e não apenas a ausência de doenças. Portanto, a pesquisa para a saúde não é limitada à estrutura e função do corpo, mas engloba fatores socioeconômicos, políticos, legais e ambientais $[6,26]$. A apresentação das etapas do processo de investigação científica, dos equipamentos utilizados e das formas de divulgação dos resultados serviu para salientar como funciona, na prática, o método científico e sua importância no processo de construção de conhecimento que busca contribuir para a melhoria da qualidade de vida da população, em especial considerando as questões osteometabólicas.

Durante a visita, as acomodações dos animais, as formas de cuidado e os parâmetros normativos seguidos na pesquisa experimental com animais foram apresentados e, simultaneamente, as questões éticas foram abordadas para que os alunos pudessem desenvolver um espírito crítico não apenas sobre o papel da pesquisa, mas também dos padrões éticos alusivos a ela. A pesquisa experimental precisa se pautar em padrões éticos tanto nos modelos experimentais em humanos quanto nos animais, buscando ir além do cuidado com normas e recomendações éticas, incentivando a discussão destes aspectos nos centros de pesquisa e nos meios de divulgação científica como eventos e periódicos $[3,28]$. Cada pesquisa tem o seu modelo experimental e deve ser planejada e executada de forma que considere o tipo de animal envolvido e a importância do respeito e da responsabilidade nesta conjuntura. Assim, deve contemplar uma análise estatística que permita utilizar o mínimo de animais para se obter resultados significativos com grau elevado de acuidade, reprodutibilidade e precisão. Além disso, deve zelar por boas condições de acomodação, nutrição e transporte, assim como pela qualidade dos procedimentos pré e pós-operatórios, incluindo a 
manipulação dos animais, a anestesia, a analgesia e a eutanásia [8, 21].

Um aspecto bastante priorizado foi a liberdade de tocar os equipamentos e manuseá-los sob orientação e supervisão. Ao mesmo tempo em que receberam informações sobre os equipamentos e suas funções, os alunos puderam averiguar as informações. Isso é importante durante o processo de aprendizagem e construção do conhecimento, pois aprender é um processo que transcende a cognição e alcança aspectos afetivos/emocionais $[17,19]$. A motivação, o estímulo, o interesse e o desejo devem ser trabalhados para que o conteúdo se torne apenas um meio de produzir conhecimento pelo prazer e pelo engajamento. Assim, o aprendizado a partir da interação promovida deve prezar as capacidades cognitivas, motriz, de relacionamento interpessoal e estratégias de ação que viabilizam o desenvolvimento de uma atividade e, assim, o indivíduo atua como agente transformador [14, 24].

As atividades práticas e dinâmicas são de extrema importância no processo de aprendizagem, pois estimulam a participação, a construção de competências e habilidades e disposições de conduta, ao contrário do que ocorre quando há apenas a transferência de informações $[1,2,15,17,19,25]$. Desta forma, a promoção de atividades de campo, fora da sala de aula e em espaços onde o assunto de interesse transcorre naturalmente, constitui uma abordagem propícia para estimular a observação, a interpretação, a análise, a visão crítica, a experimentação, a problematização e a seleção das informações mais relevantes, além de promover o interesse dos alunos sobre o assunto a ser estudado e compreendido, uma vez que o aluno se porta de maneira ativa $[5,25]$. Atividades em que os alunos de ensino básico, fundamental e médio são levados para espaços de experimentação como museus, observatórios e laboratórios constituem, portanto, um método didático pertinente neste processo [4, 10, 31, 33]. A visita ao Laboratório de Análises Morfológicas da Faculdade de Odontologia de Ribeirão Preto e ao Laboratório de Bioengenharia da Faculdade de Medicina de Ribeirão Preto, portanto, apoiou-se nessa perspectiva didática para trabalhar a compreensão dos mecanismos de investigação científica e seu papel cientifico, cultural e social, aproximando a Universidade da sociedade, especialmente alunos do ensino médio.

\section{CONCLUSÕES}

A apresentação da prática de investigação científica para alunos de ensino médio, feita de maneira didática, crítica e prática, através de visitas programadas e monitoradas ao Laboratório de Análises Morfológicas da Faculdade de Odontologia de Ribeirão Preto e ao Laboratório de Bioengenharia da Faculdade de Medicina de Ribeirão Preto foi um mecanismo para fortalecer o elo entre a universidade e a sociedade (atividade de extensão). Ao mostrar a importância das pesquisas envolvendo os distúrbios osteometabólicos para a sociedade, bem como o funcionamento do cotidiano da pesquisa e os aspectos éticos e de infraestrutura que lhes são imprescindíveis, os alunos de ensino médio puderam não apenas aprender sobre a investigação científica, mas também compreendê-la como agente transformador da realidade social. 


\section{REFERÊNCIAS}

[1] ALMEIDA, J.S.; CRUZ, J.G.; LAPA, J. M. A importância da prática na obtenção do conhecimento na educação básica, científica e tecnológica. In: VII Congresso Norte Nordeste de Pesquisa e Inovação, 2012, Palmas. Disponível em:<propi.ifto.edu.br/ocs/índex php/connepi/vii/paper/view/1760/2232>. Acesso em 3 fev. 2014.

[2] ANDRADE, M.L.F.; MASSABNI, V.G. O desenvolvimento de atividades práticas na escola: um desafio para os professores de ciências. Ciência \& Educação, Bauru, v. 17, n. 4, p. 835-854, 2011. Disponível em <http://www.scielo.br/scie-

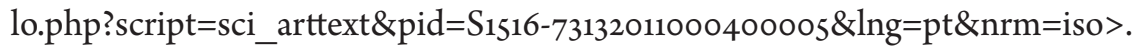
Acesso em 12 set. 2014. http://dx.doi.org/10.1590/S1516-73132011000400005.

[3] ARAUJO, L. Z. S. Aspectos éticos da pesquisa científica. Pesqui. Odontol. Bras., São Paulo, v. 17, supl. 1, maio 2003. Disponível em <http://www. scielo.br/scielo.php?script $=$ sci_arttext $\&$ pid $=$ S1517-74912003000500009\&ln $\mathrm{g}=\mathrm{pt} \& \mathrm{nrm}=\mathrm{iso}>$. Acesso em 12 set. 2014. http://dx.doi.org/10.1590/ S1517-74912003000500009.

[4] ARSLAN, L. M. et al. Conversas dentro do museu: ações educativas entre o Museu Universitário de Arte MUNA e duas escolas públicas da cidade de Uberlândia-MG. Em Extensão, Uberlândia, v.9, n.2, p. 118-123, 2010.

[5] BERNHEIM, C.T.; CHAUÍ, M. S. Desafios da Universidade na Sociedade do conhecimento: cinco anos depois da conferência mundial sobre educação superior. Brasília: UNESCO, 2008. 44p.

[6] ClarK, O. A. C.; CASTRO, A. A. A pesquisa. Pesqui. Odontol. Bras., São Paulo, v. 17, supl. 1, maio 2003. Disponível em <http://www.scielo.br/scielo.

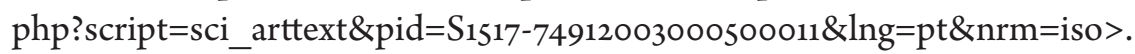
Acesso em 12 set. 2014. http://dx.doi.org/10.1590/S1517-74912003000500011.

[7] CONTO, F. A extensão universitária na faculdade de odontologia. Em Extensão, v. 12, n. 2, p. 100-108, 2013.

[8] DAMY, S.B. et. al. Aspectos fundamentais da experimentação animal - aplicações em cirurgia experimental. Rev. Ass. Med. Bras., São Paulo, v.56, n.1, p. 103-11, 2010. Disponível em <http://www.scielo.br/scielo.php?script=sci arttext\&pid=So104-42302010000100024\&lng=pt\&nrm=iso $>$. Acesso em 12 set. 2014. http://dx.doi.org/10.1590/So104-42302010000100024.

[9] DIVINO, A.E.A. et al. A extensão universitária quebrando barreiras. Cadernos de graduação - Ciências humanas e sociais, Aracaju, v. 1, n.16, p. 135-140, 2013. Disponível em: <https://periodicos.set.edu.br/index.php/cadernohumanas/ article/view/491/253>. Acesso em 16 set. 2014.

[10] FERNANDES, L. L. et al. Projeto Sábado no Campus: esportes adaptados e o goalball na formação acadêmica. Extensio, Florianópolis, n. 11, p. 32-41, 2011. Disponível em: <https://periodicos.ufsc.br/index.php/extensio/article/ view/1807-0221.2011v8n11p32/19352>. Acesso em 16 set. 2014. 
[11] FERREIRA, A.B.H. Novo Dicionário Aurélio, 2ª Edição, São Paulo: Nova Fronteira, 1986.

[12] GALIAZZI, M. C. et al. Objetivos das atividades experimentais no ensino médio: a pesquisa coletiva como modo de formação de professores de ciências. Ciênc. educ. (Bauru), Bauru, v. 7, n. 2, 2001. Disponível em <http://www. scielo.br/scielo.php?script $=$ sci_arttext\&pid $=S 1516-73132001000200008 \& \ln$ $\mathrm{g}=\mathrm{pt} \& \mathrm{nrm}=\mathrm{iso}>$. Acesso em 12 set. 2014. http://dx.doi.org/10.1590/ S1516-73132001000200008

[13] GARCIA, W. Fazer ciência: o lugar do conceito. Em Questão, Porto Alegre, v. 13, n. 1, p.171-182, 2007. Disponível em: <http://seer.ufrgs.br/index.php/EmQuestao/article/view/21>. Acesso em 16 set. 2014.

[14] GIORDAN, M. O Papel Da Experimentação no Ensino de Ciências. Química Nova na Escola, São Paulo, n. 10, 1999. Disponível em: <qnesc.sbq.org.br/ online/qnesc1o/pesquisa.pdf > . Acesso em 16 set. 2014.

[15] GOERGEN, Pedro. Ciência, sociedade e universidade. Educ. Soc., Campinas, v. 19, n. 63, ago. 1998 . Disponível em <http://www.scielo.br/scielo. php?script=sci_arttext\&pid=So101-73301998000200005\&lng=pt\&nrm=iso $>$. Acesso em 12 set. 2014. http://dx.doi.org/10.1590/So101-73301998000200005.

[16] KOSMINSKY, L.; GIORDAN, M. Visões sobre Ciências e sobre o Cientista entre Estudantes do Ensino Médio. Química Nova na Escola, São Paulo, n. 15, p. 11-18, 2002. Disponível em: <qnesc.sbq.org.br/online/qnesc15/v15a03.pdf>. Acesso em 16 set. 2014.

[17] LIRA, L.S. A Importância da Prática Experimental no Ensino de Biologia na Educação de Jovens e Adultos. João Pessoa, 2013, 65p. Monografia, Centro de Ciências Exatas e da Natureza, Universidade Federal da Paraíba.

[18] LONGO, W.P. Alguns impactos sociais do desenvolvimento científico e Tecnológico. Edu.Tec, ano I, v.1, n.1, 2008. Disponível em:<www.faetec.rj.gov.br >. Acesso em: 28 out. 2013.

[19] MARSULO, M.A.G.; SILVA, R.M.G. Os métodos científicos como possibilidade de construção de conhecimentos no ensino de ciências. Revista Electrónica de Enseñanza de las Ciencias, Pondevedra, v. 4, n.3, p. 1-12, 2005. Disponível em: <reec.uvigo.es/volumenes/volumen4/ART3_Vol4_N3.pdf>. Acesso em 16 set. 2014.

[20] MARTINS, J.S. O trabalho com projetos de pesquisa: do ensino fundamental ao ensino médio, $5^{\text {a }}$. Edição. Campinas: PAPIRUS, 2001.

[21] MIZIARA, I. D. et al . Research ethics in animal models. Braz. j. otorhinolaryngol., São Paulo, v. 78, n. 2, abr. 2012 . Disponível em <http://www. scielo.br/scielo.php? script $=$ sci_arttext\&pid $=$ S1808-86942012000200020\&ln $\mathrm{g}=\mathrm{pt} \& \mathrm{nrm}=\mathrm{iso}>$. Acesso em 12 set. 2014. http://dx.doi.org/10.1590/ S1808-86942012000200020.

[22] NASCIMENTO, I. R.T. A Indissociabilidade entre Pesquisa e Extensão nas Universidades: O Caso da ITES/UFBA. Revista NAU Social, v.3, n.5, p. 4146, 2013. Disponível em: <www.periodicos.adm.ufba.br/index.php/rs/article/ viewFile/244/193>. Acesso em 16 set. 2014. 
[23] NUNES, A.L.P.F.; SILVA, M.B.C. A extensão universitária no ensino superior e a sociedade. Mal-Estar e Sociedade, Barbacena, ano IV, n. 7, p. 119-133, 2011. Disponível em: <www.uemg.br/openjournal/index.php/malestar/article/ viewFile/6o/89>. Acesso em 16 set. 2014.

[24] PEREIRA, D. S. C. O ato de aprender e o sujeito que aprende. Constr. psicopedag., São Paulo, v. 18, n. 16, jun. 2010. Disponível em <http://pepsic.bvsalud.org/scielo.php?script $=$ sci_arttext\&pid $=S 1415-69542010000100010 \& \operatorname{lng}=$ pt\&nrm=iso $>$. Acesso em 12 set. 2014.

[25] PINTO, H. P. P.; PIERUCCI, A. Meu corpo, minha fortaleza: uma relação entre anatomia humana e saúde. Em Extensão, v. 12, n. 1, p. 174-185, 2013.

[26] ROBERTO, C.K.M.; SABINO, G.A. A importância da Pesquisa Científica na Área da Saúde. Revista Movimenta, v. 2, n.4, 2009. Disponível em: <nee. ueg.br/seer/index.php/movimenta/article/viewFile/303/253>. Acesso em 16 set. 2014.

[27] SAWADA, N. O.; PEDRAZZI, V.; RODRIGUES, M. L. V. I Fórum de Integração dos Serviços de Saúde e das Atividades de Ensino, Pesquisa e Extensão da USP. Revista de Cultura e Extensão USP, Brasil, v. 6, p. 33-40, out. 2011. ISSN 2316-9060. Disponível em: <http://www.revistas.usp.br/rce/article/view/532>. Acesso em 12 Set. 2014. http://dx.doi.org/10.116o6/issn.23169060.v6iop33-40.

[28] SCHNAIDER, T. B. Ética e pesquisa. Acta Cir. Bras., São Paulo, v. 23, n. 1, fev. 2008. Disponível em <http://www.scielo.br/scielo.php?script=sci arttext\&pid=So102-86502008000100017\&lng=pt\&nrm=iso $>$. Acesso em 12 set. 2014. http://dx.doi.org/10.1590/So102-86502008000100017.

[29] SILVA, R.N. Importância, desafios e perspectivas da extensão universitária. Em Extensão, v. 10, n. 2, p. 204-206, 2011.

[30] SOARES, L.R.; FARIAS, M.C.M.; FARIAS, M.M. Ensino, pesquisa e extensão: histórico, abordagens, conceitos e Considerações. Em Extensão, v.9, n.1, p. 11$18,2010$.

[31] SOUZA, R.R. Uma aula diferente de climatologia. Em Extensão, v.9, n.2, p.185192, 2010.

[32] VIEIRA, J. G. H. Diagnóstico laboratorial e monitoramento das doenças osteometabólicas. J. Bras. Patol. Med. Lab., Rio de Janeiro, v. 43, n. 2, abr. 2007. Disponível em <http://www.scielo.br/scielo.php?script=sci_arttext\&pid=S1676$-24442007000200002 \& \operatorname{lng}=p t \& n r m=i s o>$. Acesso em 12 set. 2014. http:// dx.doi.org/10.1590/S1676-24442007000200002.

[33] VIVEIRO, A. A.; DINIZ, R.E.S. As atividades de campo no ensino de ciências: reflexões a partir das perspectivas de um grupo de professores. In: NARDI, R. Ensino de ciências e matemática, I: temas sobre a formação de professores. São Paulo: Editora UNESP; São Paulo: Cultura Acadêmica, 2009. Cap. 2, p. 27-42. 
TAIANE PALIOLOgO graduanda do curso de Fisioterapia na Faculdade de Medicina de Ribeirão Preto da Universidade de São Paulo (FMRP-USP)

MILIANE GONÇALVES GONZAGA mestranda na Faculdade de Medicina de Ribeirão Preto da Universidade de São Paulo (FMRP-USP)

VINícIUS PEDRAZZI professor associado 3 na Faculdade de Odontologia de Ribeirão Preto da Universidade de São Paulo (FORP-USP)

JOÃO PAULO MARDEGAN ISSA professor associado 1 na Faculdade de Odontologia de Ribeirão Preto da Universidade de São Paulo (FORP-USP) - e-mail: jpmissa@forp.usp.br 